\title{
Solvent-Controlled Morphology of Catalytic Monolayers at Solid-Liquid Interfaces
}

\author{
Kraack, Jan Philip ; Hamm, Peter
}

\begin{abstract}
Ultrafast vibrational dynamics of monolayers from a rhenium carbonyl complex at solid-liquid interfaces is investigated with the help of 2 D ATR IR spectroscopy in dependence of the solvation environment. By changing the solvent from methanol to acetonitrile, we observe a structural transition between two states and find that this transition is fully reversible. The two coexisting states are identified by distinct but closely spaced $(15 \mathrm{~cm}-1)$ vibrational bands in the region of the A (1) symmetric carbonyl stretch vibration. Surface-dilution experiments suggest that the newly formed state in acetonitrile is related to aggregation of molecules at the surface. The vibrational energy transfer rate between the two species ( $30 \mathrm{ps}$ ) is used as a structural measure, based on which the morphology is characterized as partially ordered dimers in an otherwise disordered but closely packed monolayer.
\end{abstract}

DOI: https://doi.org/10.1021/acs.jpcc.7b12421

Posted at the Zurich Open Repository and Archive, University of Zurich

ZORA URL: https://doi.org/10.5167/uzh-150195

Journal Article

Accepted Version

Originally published at:

Kraack, Jan Philip; Hamm, Peter (2018). Solvent-Controlled Morphology of Catalytic Monolayers at Solid-Liquid Interfaces. Journal of Physical Chemistry C, 122(4):2259-2267.

DOI: https://doi.org/10.1021/acs.jpcc.7b12421 


\title{
Solvent-Controlled Morphology of Catalytic Monolayers at Solid-Liquid Interfaces
}

\author{
Jan Philip Kraack*, and Peter Hamm ${ }^{\ddagger}$ \\ Department of Chemistry, University of Zurich, Winterthurerstrasse 190, CH-8057
}

\begin{abstract}
Ultrafast vibrational dynamics of monolayers from a Rhenium carbonyl complex at solid-liquid interfaces are investigated with the help of 2D ATR IR spectroscopy in dependence of the solvation environment. By changing the solvent from methanol to acetonitrile, we observe a structural transition between two states, and find that this transition is fully reversible. The two co-existing states are identified by distinct but closely spaced $\left(\sim 15 \mathrm{~cm}^{-1}\right)$ vibrational bands in the region of the $A^{\prime}(1)$ symmetric carbonyl stretch vibration. Surface-dilution experiments suggest that the newly formed state in acetonitrile is related to aggregation of molecules at the surface. The vibrational energy transfer rate between the two species $(\sim 30 \mathrm{ps})$ is used as a structural measure, based on which the monolayer morphology is characterized as partially ordered dimers in an otherwise disordered but closely packed monolayer.
\end{abstract}




\section{Introduction}

Heterogeneous catalysis at solid-liquid interfaces is a central research direction in current chemical physics and technology. ${ }^{1-7}$ Particularly, the molecular structure of catalytically-active species as well as reactants and reaction products are of paramount importance. A central reaction in heterogeneous catalysis is represented by the conversion of $\mathrm{CO}_{2}$ to usable products. ${ }^{8-16}$ In heterogeneous environments, the catalysts are generally covalently attached to surfaces. But very often there exists a lack of knowledge regarding detailed mechanisms of adsorbate-adsorbate and adsorbate-environment interactions. In this regard, the distances between different species at the surface is an important parameter and it is often quite short, i.e. a few Angströms. ${ }^{17-19}$ Such short distances therefore may be thought to result in extensive interactions between the different molecules. ${ }^{17,20}$ In typical heterogeneous samples, the exact morphological structure at the interface is generally not known, and it is thus not clear whether the molecules behave as independent entities, or if cooperative effects occur. Of particular importance in this respect is the formation of aggregates at the surface. ${ }^{21-25}$ Particularly in the case of active $\mathrm{CO}_{2}$ reduction catalysts, the formation of such aggregates, or even covalent binding between two molecules, has been proposed to be an essential step in the catalytic cycle. ${ }^{23-25}$ In the context of dye-sensitized solar cells, dye aggregates on the semiconductor surface are believed to limit their efficiency. ${ }^{26,27}$

Here, we demonstrate that the morphology of a monolayer of a Rhenium carbonyl catalysts at semiconductor-liquid interfaces can be controlled to a certain extent by the choice of the solvent, allowing us to switch reversibly between a closely packed but disordered monolayer and a partially ordered one, the latter of which containing dimerized catalyst molecules. By use of 2D ATR IR spectroscopy, ${ }^{18,28-33}$ we can structurally characterize these monolayers. 2D ATR IR is a relatively new method for surface vibrational spectroscopy with sub-picosecond temporal resolution, which we have introduced before. ${ }^{28,34}$ Similar as standard 2D IR spectroscopy ${ }^{35-38}, 2 \mathrm{D}$ ATR IR is based on the third-order nonlinear response of the sample. However, 2D ATR IR uses evanescent waves generated at the interface between two materials of a high and a low refractive index. ${ }^{39-41} 2 \mathrm{D}$ ATR IR is sensitive to all molecules within the penetration-depth of the evanescent wave, which may extend to several micrometres off the interface and obtaining surface-sensitive signals requires immobilization of the molecules. ${ }^{39-41}$ We have recently investigated with the help of 2D ATR IR the ultrafast dynamics of for instance surface-bound diatomics ${ }^{28,31}$ or organic monolayers. ${ }^{29,30}$

The sample under investigation in the present study is $\operatorname{Re}\left(4,4^{\prime}\right.$-dicarboxyl-2,2'-bipyridine $)(\mathrm{CO})_{3} \mathrm{Cl}$ $\left(\operatorname{Re}(\mathrm{CO})_{3} \mathrm{Cl}\right.$, Figure 1), which can perform as a catalyst for $\mathrm{CO}_{2}$-reduction. ${ }^{23,42}$ Here, we focus on the ultrafast vibrational dynamics of the symmetric stretching vibration $\left(A^{\prime}(1), \sim 2020-2040 \mathrm{~cm}^{-1}\right.$, Figure 1, red shaded region) of the carbonyl ligands from the surface-bound catalysts. ${ }^{19,23,43-45}$ Two other carbonyl 
stretching modes $\left(A^{\prime}(2)\right.$ and $\left.A^{\prime \prime}\right)$ are spectrally located outside the present observation window. We consider here monolayers of $\operatorname{Re}(\mathrm{CO})_{3} \mathrm{Cl}$ immobilized on a indium-tin-oxide (ITO) surface, that is deposited on the reflecting plane of a $\mathrm{CaF}_{2}$ ATR prism (we consider ITO layers since they may serve as electrodes for ATR spectro-electrochemistry $\left.{ }^{31}\right)$. The catalyst molecules are bound via two carboxylate functional groups to the oxide vacancies of an ITO surface (Figure 1).

2D IR spectra of this or very similar molecules on oxide surfaces (mostly $\mathrm{TiO}_{2}$ and silica) have been measured numerous times, revealing that the IR spectroscopy is surprisingly complex. ${ }^{18,19,21,22,44,46,47}$ For example, Zanni and co-workers observed multiple peaks in the symmetric stretching region of basically the same molecule on $\mathrm{TiO}_{2}$, which they first assigned to heterogeneous binding sites, ${ }^{46}$ and later to the formation of dimers as well as larger aggregates. ${ }^{21,22}$ Fayer and co-workers studied a very similar Recomplex, albeit with a longer linker, on metal surfaces, where the main peak was accompanied by a small shoulder on the high frequency side, the origin of which, however, remained elusive. ${ }^{44}$ Very recently, the same group studied the dynamics of the same molecule in monolayers of LangmuirBlodgett films, again observing complex IR spectra which evolve on a very slow second to hour timescales. ${ }^{48}$ In the present paper, we also aim to clarify some of the confusion that exists about the IR spectroscopy of this important molecule on surfaces.

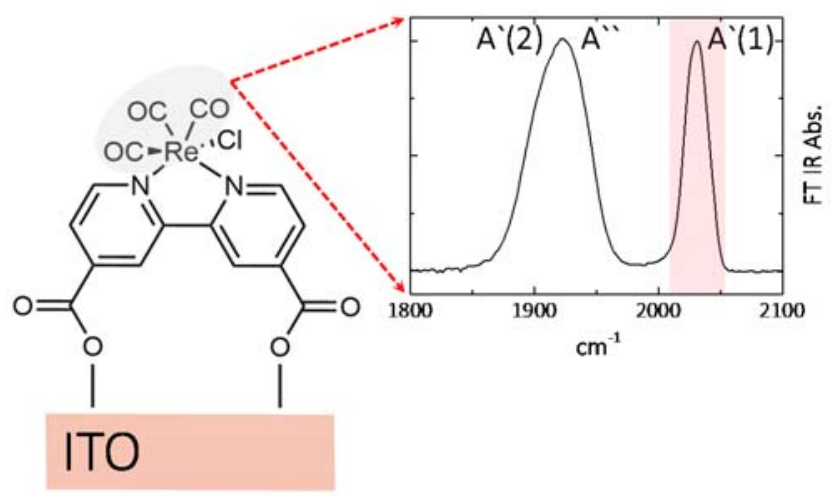

Figure 1. Sample molecule ( $\operatorname{Re}\left(4,4^{\prime}\right.$-dicarboxyl-2,2'-bipyridine $)(\mathrm{CO})_{3} \mathrm{Cl}$, abbreviated as $\left(\operatorname{Re}(\mathrm{CO})_{3} \mathrm{Cl}\right)$ immobilized on an Indium-Tin-Oxide (ITO) surface. The three $\mathrm{CO}$ ligands have three IR absorption bands ( $A^{\prime}(1), A^{\prime}(2)$, and $\left.A^{\prime \prime}\right)$ in the spectral range $1800-2100 \mathrm{~cm}^{-1}$; the present study focuses on the $A^{\prime}(1)$ mode (red shaded region).

\section{Materials and Methods}

A detailed description of our experimental setup for 2D ATR IR spectroscopy has been given previously. ${ }^{28,34}$ In brief, the spectrally tunable output of an optical parametric amplifier (OPA, 100 fs) with a $5 \mathrm{kHz}$ repetition rate is split into pump, probe and reference beams by help of a $\mathrm{BaF}_{2}$ wedge. Coherent pump pulse pairs for 2D spectroscopy are generated by help of a Mach-Zehnder 
interferometer. ${ }^{49}$ The three beams are focused onto the reflecting plane of a $\mathrm{CaF}_{2}$ ATR prism, spatially separated behind the sample and probe/reference beams are balance-detected with a mercury cadmium telluride (MCT) array detector. All measurements have been performed with s-polarized light to prevent the formation of evanescent waves with elliptical polarization at the interface. ${ }^{32}$

$\mathrm{CaF}_{2}$ prisms (Thorlabs) were sputter-coated with ITO layers (5 nm average thickness) in a Bal-tec SCD 500 sputter coater (Leica Microsystems, Vienna, Austria). The thickness of the ITO layers is determined in-situ with a quartz microbalance. Sputtering was carried out at a working distance of $50 \mathrm{~mm}$, a pressure of $0.8 \times 10^{-5} \mathrm{mbar}$, an Ar pressure of $0.1 \mathrm{mbar}$, and a current of $30 \mathrm{~mA}$, resulting in a deposition rate of about $0.02 \mathrm{~nm} \mathrm{~s}^{-1}$. $\operatorname{Re}\left(4,4^{\prime}\right.$-dicarboxyl-2,2'-bipyridine $)(\mathrm{CO})_{3} \mathrm{Cl}\left(\operatorname{Re}(\mathrm{CO})_{3} \mathrm{Cl}\right)$ was synthesized as described earlier. ${ }^{18}$ The starting materials $\mathrm{Re}(\mathrm{CO})_{5} \mathrm{Cl}$ and 2,2'-bipyridine-4,4'-dicarboxylic acid were purchased from Sigma Aldrich and used without further purification. Methanol and acetonitrile (spectroscopic-grade) were purchased from Sigma-Aldrich and used without further purification. For the preparation of the monolayers, $\operatorname{Re}(\mathrm{CO})_{3} \mathrm{Cl}$ was dissolved in methanol $(\mathrm{MeOH})$ to a concentration of $0.1 \mathrm{mM}$. The freshly coated prisms were immersed overnight in that solution, and afterwards thoroughly washed with $\mathrm{MeOH}$ and sonicated. For a solvent exchange, the samples was first dried and re-immersed in the corresponding solvent (typically overnight).

\section{Results and Discussion}

\section{1 Solvent-Control of the Monolayer}

2D ATR IR spectra of a $\operatorname{Re}(\mathrm{CO})_{3} \mathrm{Cl}$ monolayer on an ITO surface are shown in Figure 2 for different experimental conditions. When the sample was prepared from a $\operatorname{Re}(\mathrm{CO})_{3} \mathrm{Cl}$ solution in $\mathrm{MeOH}$, and subsequently kept in neat $\mathrm{MeOH}$ in the ATR sample cell, the 2D ATR IR signal exhibits the usual ground state bleach/stimulated emission contributions (GSB/SE, blue) from a single band at $2025 \mathrm{~cm}^{-1}$ in the spectral region of the symmetric carbonyl stretch vibration (Figure $2(a)$ ), along with the corresponding excited state absorption band (ESA, red). Both GSB/SE and ESA features appear strongly elongated along the diagonal line, which is indicative for strong inhomogeneous broadening of the sample. ${ }^{35,50}$ The heterogeneity is based on the different local environments of the molecules attached to the heterogeneous ITO surface. ${ }^{18}$ By re-immersing the same sample in-situ in acetonitrile (MeCN), a second band appears at about $2040 \mathrm{~cm}^{-1}$ after about $2 \mathrm{~h}$ (Figure 2 (b)), again consisting of a GSB/SE and a ESA signal. The second peak continues to grow in intensity by overnight treatment ( $\sim 16 \mathrm{~h}$ ) with MeCN (Figure 2 (c)). It is important to note that the second band does not increase any further beyond that point, even for MeCN treatment over days. The experimental data thus indicate that MeCN induces some sort of transition in the monolayer, characterized by a higher-frequency band, but that second form, which is observed only in MeCN, exists in an equilibrium with the original one. It is possible that 
this second peak is the related to the shoulder observed by Fayer and co-workers in Ref. ${ }^{44}$, who also worked in MeCN but presumably used a fresh sample.
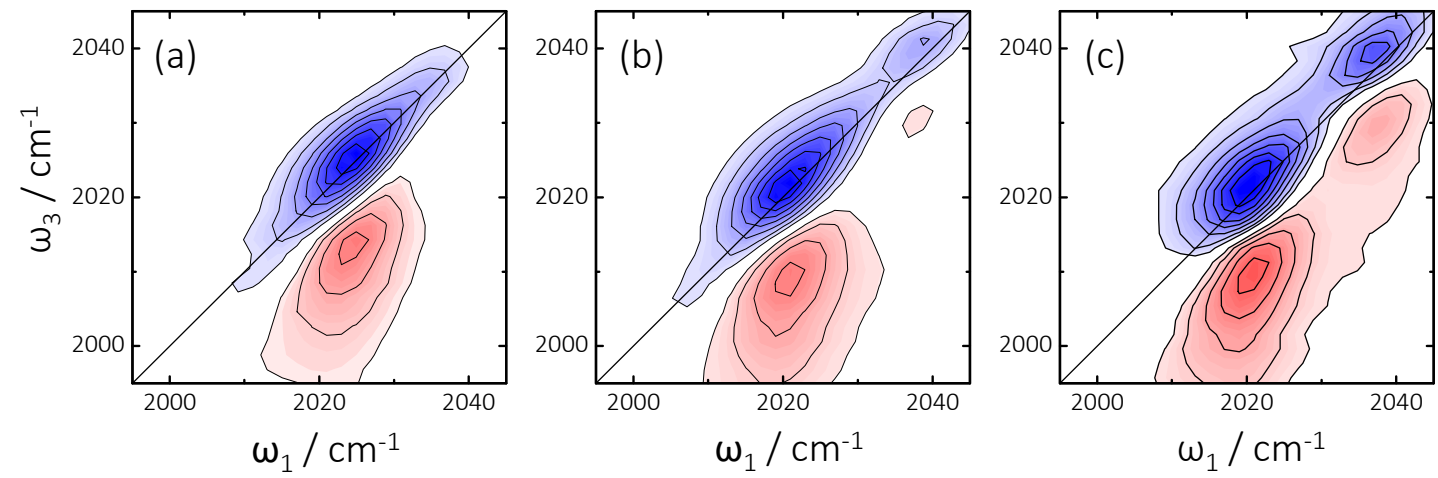

Figure 2. 2D ATR IR spectra of the $\mathrm{Re}(\mathrm{CO})_{3} \mathrm{Cl}$ monolayers on $5 \mathrm{~nm}$ ITO surfaces under different experimental conditions. (a) A pure $\mathrm{Re}(\mathrm{CO})_{3} \mathrm{Cl}$ sample, immersed in methanol (MeOH). (b) and (c) The same sample following in-situ treatment with acetonitrile (MeCN) after (b) about $2 \mathrm{~h}$, and (c) overnight incubation ( $16 \mathrm{~h}$ ). All 2D ATR IR have been measured at a population time of 0.25 ps.

Interestingly, that transition is fully reversible, as demonstrated in Figure 3, where we show two cycles of alternating solvent treatments of one and the same sample with $\mathrm{MeCN}$ and $\mathrm{MeOH}$. That is, if a sample, which has first been treated in $\mathrm{MeCN}$ (Figure 3b), is re-immersed in $\mathrm{MeOH}$, the second band at $2040 \mathrm{~cm}^{-1}$ disappears, leading again to a single resonance at about $2025 \mathrm{~cm}^{-1}$ (Figure 3 (c)), similar to the one of the original $\mathrm{MeOH}$ sample (Figure 3 (a)). Subsequent incubation in MeCN causes the $2040 \mathrm{~cm}^{-1}$ band to re-appear (Figure 3 (d)). 

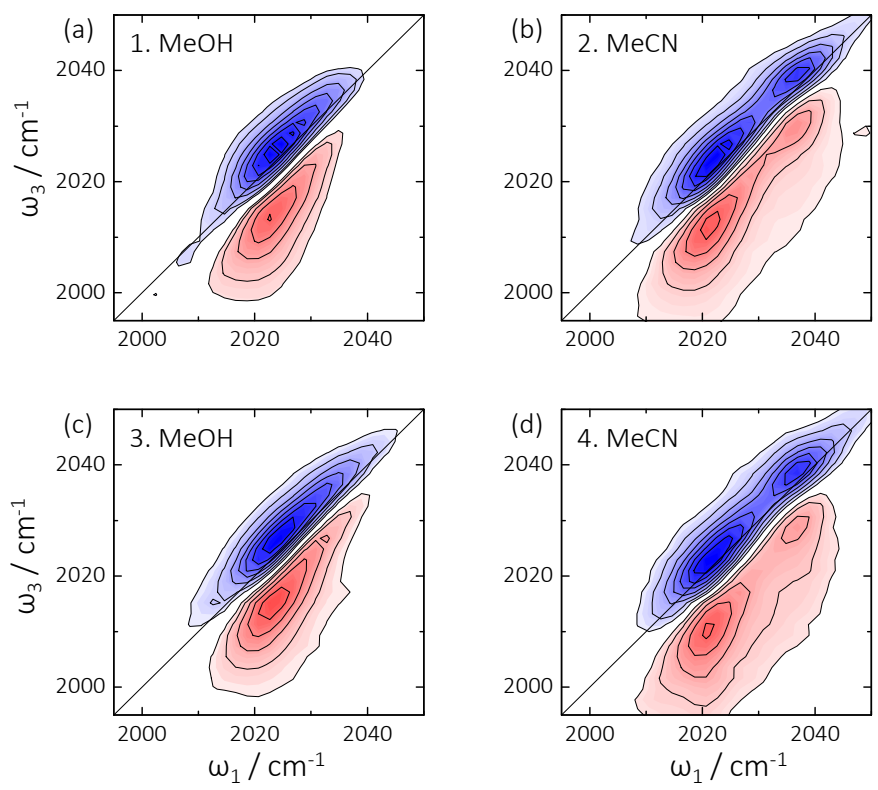

Figure 3. Reversible generation of double band structures in a $\operatorname{Re}(\mathrm{CO})_{3} \mathrm{Cl}$ monolayer on $5 \mathrm{~nm}$ thick ITO surfaces. (a) Sample prepared from $\mathrm{MeOH}$ solution of the $\mathrm{Re}(\mathrm{CO})_{3} \mathrm{Cl}$ and kept in $\mathrm{MeOH}$ environment during the measurement. (b) Sample taken from (a), re-immersed in $\mathrm{MeCN}$ overnight and measured in MeCN environment. (c) Sample taken from (b), re-immersed again in $\mathrm{MeOH}$ overnight and measured in $\mathrm{MeOH}$ environment. (d) Sample taken from (c), reimmersed again in MeCN overnight and measured in MeCN environment.

We can think of three possible explanations for the double peak structure appearing in MeCN environment, out of which we favor the one proposed recently by Zanni and coworkers. ${ }^{21,22}$ Based on stationary 2D IR and FTIR experiments as well as on quantum-chemical calculations, they have suggested that aggregation of $\operatorname{Re}(\mathrm{CO})_{3} \mathrm{Cl}$ molecules at semiconductor surfaces results in the generation of numerous vibrational bands on the high-frequency side of the monomer band under certain experimental conditions. ${ }^{21,22}$ In comparison to their spectra, ours are in fact less complex with only two bands, which we therefore assign to monomeric $\operatorname{Re}(\mathrm{CO})_{3} \mathrm{Cl}$ molecule $\left(2025 \mathrm{~cm}^{-1}\right)$ vs. dimers $\left(2040 \mathrm{~cm}^{-1}\right.$ , we will hereafter use the term of dimerization instead of aggregation). As alternative explanations, the two bands might originate from different binding sites on the surface or from exchange of the chloride ligand in the coordination sphere of the Rhenium center with a MeCN molecule. In the following, we will discuss the results of Figure 2 and Figure 3 in the context of Zanni's model, and will furthermore present various additional experiments done to obtain a better understanding of the morphology of these monolayers. In addition in Sec. III.3, we will present a couple of control experiments done to exclude those alternative explanations.

We start with a surface dilution experiment by introducing "dilutant" molecules, which are intended to block surface adsorption sites. This is done by adding different amounts of benzoic acid (Ph) to the 
$\operatorname{Re}(\mathrm{CO})_{3} \mathrm{Cl}$ solution. The result is the formation of a series of mixed monolayer samples with different ratios of $\mathrm{Ph}$ vs. $\operatorname{Re}(\mathrm{CO})_{3} \mathrm{Cl}$. Measuring the $\mathrm{CO}$ signal magnitude of the mixed $\mathrm{Ph} / \operatorname{Re}(\mathrm{CO})_{3} \mathrm{Cl}$ layers relative to a pure $\operatorname{Re}(\mathrm{CO})_{3} \mathrm{Cl}$ monolayer under otherwise identical conditions gives an estimate of the surface dilution of $\mathrm{Re}(\mathrm{CO})_{3} \mathrm{Cl}$. Figure 4 shows $2 \mathrm{D}$ ATR IR spectra for surface dilution ratios from $40 \%$ down to $16 \%$ for samples prepared from $\mathrm{MeOH}$ solutions ((a), (c)), as well as after overnight treatment of the same samples with MeCN ((b), (d)). Both samples initially exhibit the same $2025 \mathrm{~cm}^{-1}$ band in $\mathrm{MeOH}$, whereas the $2040 \mathrm{~cm}^{-1}$ band, which is still visible at mild dilution (40\%, (b)), disappears when going to strong dilution $(16 \%,(d))$. Interfacial dimerization is a bimolecular process, and as such, the equilibrium of monomer vs. dimer is expected to depend on surface concentration. The result of Figure 4 thus strongly supports the dimerization model of Zanni and coworkers ${ }^{21,22}$, where the relative contribution of the $2040 \mathrm{~cm}^{-1}$ band gradually reduces upon dilution, until it completely vanishes at highest dilution $(16 \%,(d))$.
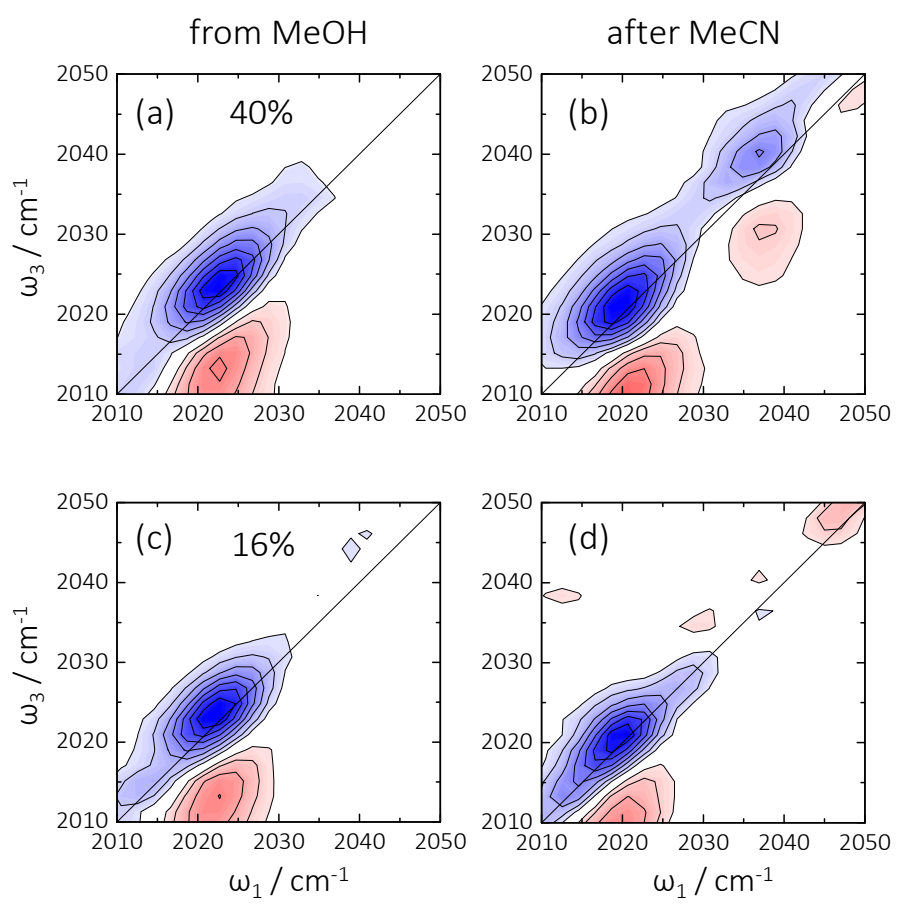

Figure 4. 2D ATR IR spectra of mixed monolayers $\left(\mathrm{Ph} / \mathrm{Re}(\mathrm{CO})_{3} \mathrm{Cl}\right)$ of benzoic acid $(\mathrm{Ph})$ and $\mathrm{Re}(\mathrm{CO})_{3} \mathrm{Cl}$ on ITO surfaces. (a) and (b) for $40 \%$ surface coverage and incubation with $\mathrm{MeOH}$ and $\mathrm{MeCN}$, respectively, and (c) and (d) the same for $16 \%$ surface coverage. The spectra are normalized to the $2025 \mathrm{~cm}^{-1}$ band and measured at a population time of $0.25 \mathrm{ps}$.

\section{2 Morphology of the Monolayer}

One particular strength of 2D IR methods is its capability to determine intermolecular interactions, e.g. the identification of vibrational energy transfer with sub-picosecond temporal resolution. ${ }^{34}$ In particular, 
2D ATR IR has recently been used to characterize vibrational energy transfer dynamics in $\operatorname{Re}(\mathrm{CO})_{3} \mathrm{Cl}$ monolayers on ITO surfaces by help of isotope labelling experiments. ${ }^{18}$ As a central point, that study determined the intermolecular distances within the monolayer as a structural parameter. Building up on that work, we conduct here time-dependent 2D ATR IR measurements to investigate the interactions between the two forms of surface-bound $\mathrm{Re}(\mathrm{CO})_{3} \mathrm{Cl}$ in $\mathrm{MeCN}$ environment. We start with noting that no cross peaks between the symmetric stretch vibrations of the two bands are present in the earlypopulation time 2D ATR IR spectra shown in of Figure 2 (b) and (c), which would indicate strong coupling and mixing of the states. In the limit of weak dipole-dipole coupling, vibrational energy transfer may still occur, giving rise to cross peaks that grow in as a function of population time. ${ }^{18,35}$ To explore that possibility, Figure 5 shows a series of 2D ATR IR spectra for a fully covered surface of $\operatorname{Re}(\mathrm{CO})_{3} \mathrm{Cl}$ on ITO, where indeed distinct cross peaks show up above and below the diagonal after a few picoseconds. We note in passing that such time-dependent cross peaks may in principle also originate from chemical exchange $e^{48,51,52}$, i.e. interconversion of the two species. However, that possibility can be safely ruled out here, since we know already from Figure 2 that such an exchange takes place on an hour timescale and not on a picosecond timescale.
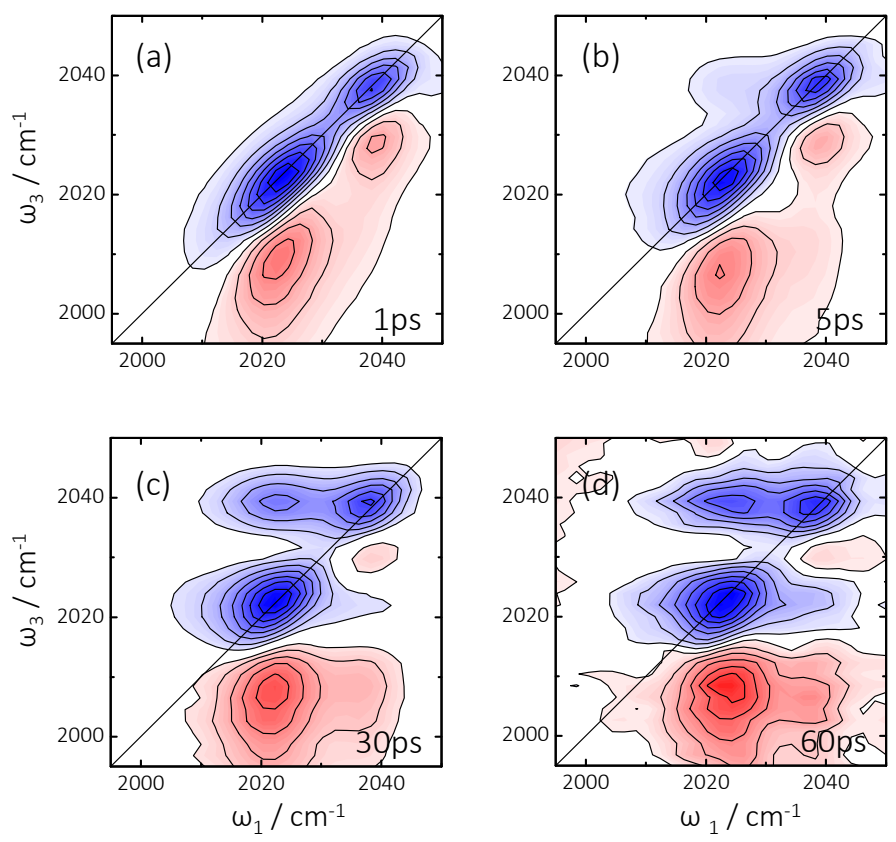

Figure 5. 2D ATR IR spectra of $\mathrm{Re}(\mathrm{CO})_{3} \mathrm{Cl}$ on $5 \mathrm{~nm}$ ITO surfaces on a $\mathrm{CaF}_{2}$ prism after MeCN treatment. Blue/red signals correspond to GSB/SE and ESA contributions, respectively. Cross peaks between the two different bands appear with increasing population delays. (a) 1 ps population delay, (b) $5 \mathrm{ps}$, (c) $30 \mathrm{ps,} \mathrm{(d)} 60 \mathrm{ps}$. The spectra are normalized to the $2025 \mathrm{~cm}^{-1}$ diagonal band. 
For a quantitative analysis of the energy transfer giving rise to the cross peaks, we analyse the intensity of the diagonal and cross peak features in dependence of the population time (Figure 6 (a)). We focus on the GSB/SE signal of the diagonal peak at $2025 \mathrm{~cm}^{-1}$ as well as the GSB/SE of the cross peak at the upper diagonal position, since the latter contribution is not affected in intensity by cancellation effects with one of the diagonal peaks. The diagonal GSB/SE signal (black open squares) decays monotonically. The signal at the cross peak position (red open circles) exhibits an initial decay until about 1 ps, increases afterwards, runs through a maximum at about 15 ps and eventually decays again. The initial decay is due to contributions from the tail of the intense GSB/SE diagonal peak and thus is not related to the direct cross peak dynamics. To extract time constants for vibrational relaxation as well as energy transfer between the two bands, we fitted the experimental data by a kinetic model, which assumes that both species $\left(2025 \mathrm{~cm}^{-1}, 2040 \mathrm{~cm}^{-1}\right)$ can be independently excited (stars) and afterwards undergo either

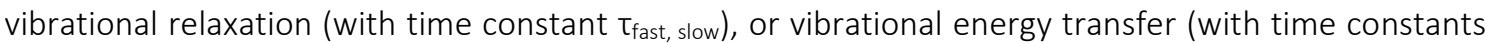
$\tau_{E T}$, bw/fw, where bw $\equiv$ backward and $f w \equiv$ forward, see Figure 6 (b)). We note that a bi-exponential vibrational relaxation with a fast and a slow component has often been observed for Rhenium tricarbonyl complexes and is tentatively attributed to intramolecular vibrational energy redistribution between the $A^{\prime}$ and $A^{\prime \prime}$ modes of the complex, ${ }^{53-56}$ but surface heterogeneity might contribute as well. The fit with this model describes the experimental data very well and yields time constants for vibrational relaxation of $\tau_{\text {fast }}=2 \mathrm{ps}$ and $\tau_{\text {slow }}=25 \mathrm{ps}$ with amplitude ratios of $0.3 / 0.7$, respectively, as well as $\tau_{\mathrm{ET} \text {, bw }}=34 \mathrm{ps}$ and $\tau_{\mathrm{ET}, \mathrm{fw}}=32 \mathrm{ps}$. While the time constants of vibrational relaxation are very close to previous observations ${ }^{18,43,53}$, vibrational energy transfer is by about a factor three faster than in our previous report ( $\sim 90 \mathrm{ps}){ }^{18}$ 

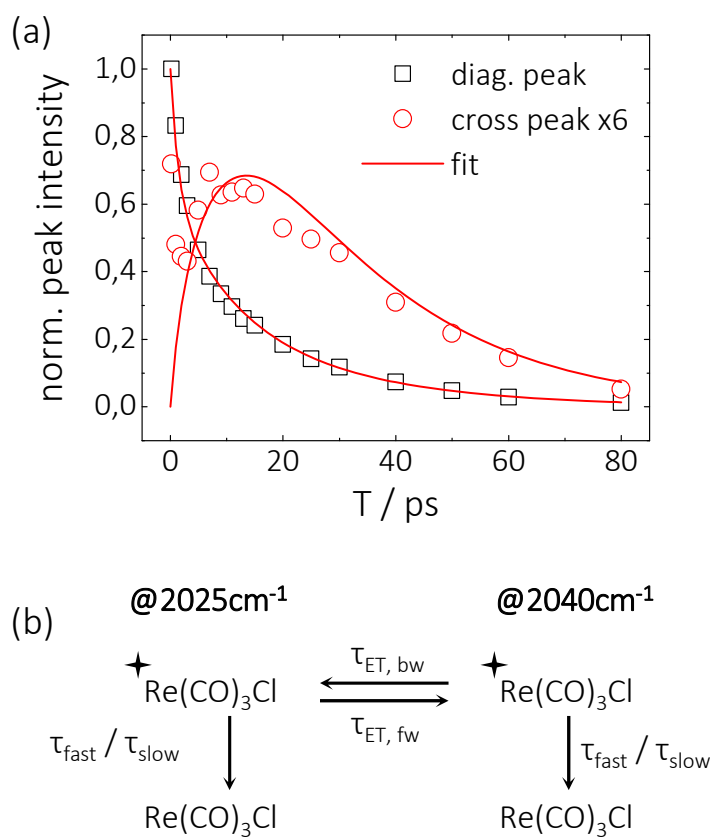

Figure 6. (a) Experimental data for diagonal signals $\left(2025 \mathrm{~cm}^{-1}\right.$, open black squares) and cross peak signals ( $2040 \mathrm{~cm}^{-1}$, open red circles) together with a fit described in the text (red lines). The cross peak intensity has been magnified by a factor of six. (b) Employed model for exponential fitting. $\tau_{\text {fast, slow }}$ are time constants for vibrational relaxation whereas $\tau_{E T}$, bw/fw are time constants for energy transfer, where bw $\equiv$ backward and fw $\equiv$ forward.

It is furthermore instructive to investigate energy transfer for samples with reduced surface coverage of $\operatorname{Re}(\mathrm{CO})_{3} \mathrm{Cl}$. Figure 7 shows a time series of $2 \mathrm{D}$ ATR IR spectra of a sample with the $\operatorname{Re}(\mathrm{CO})_{3} \mathrm{Cl}$ surface coverage reduced to $40 \%$. Both bands at $2025 \mathrm{~cm}^{-1}$ and $2040 \mathrm{~cm}^{-1}$ are still present at this level of dilution (see also Figure 4 (b)), hence dimerization still occurs, yet no cross peaks can be detected up to population delays of $30 \mathrm{ps}$ (when the cross peak are already clearly visible in the fully coverage sample of Figure $5(\mathrm{c})$ ), indicating that vibrational energy transfer is markedly suppressed. 

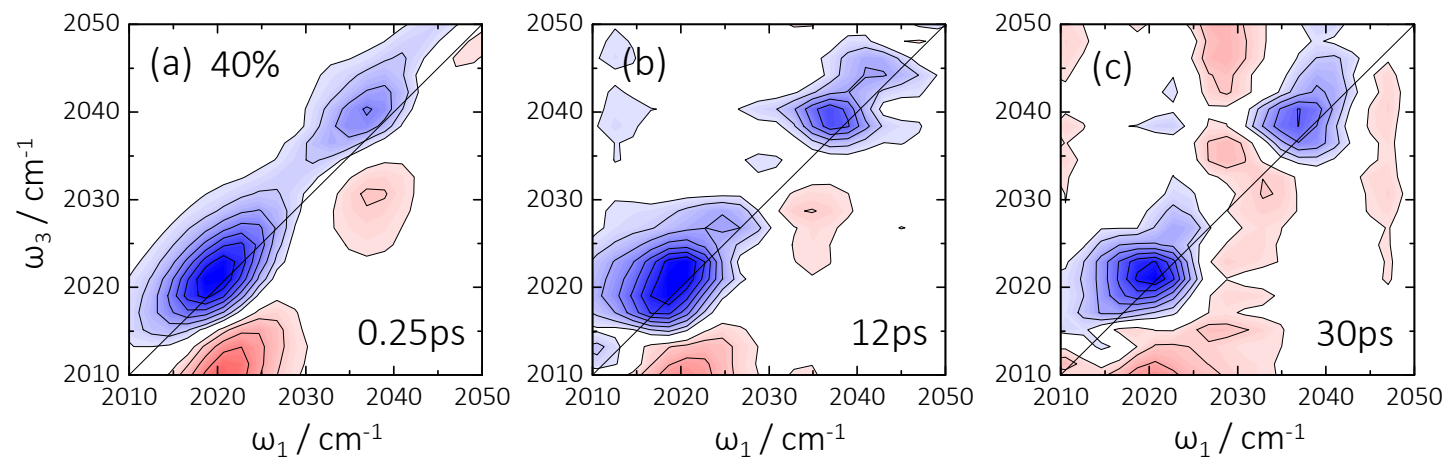

Figure 7. Time series of 2D ATR IR spectra of mixed monolayers on ITO surfaces for a surface coverage of $40 \%$. No cross peaks exist between the two peaks, indicating that the dimers are spatially separated from the monomers. (a) 0.25 ps, (b) 12 ps, (c) 30 ps population delay. The spectra are normalized to the $2025 \mathrm{~cm}^{-1}$ diagonal band.

In close analogy to NOESY in 2D NMR or Förster energy transfer in electronic fluorescence spectroscopy, ${ }^{57-65}$ the vibrational energy transfer rate $k_{D A}$ can be described by: ${ }^{64}$

$$
\frac{1}{\tau_{D A}}=\mathrm{k}_{\mathrm{DA}}=\frac{2}{1+\exp \left(\frac{-\Delta \omega}{k_{B} T}\right)} V_{D A}^{2} \frac{T_{2}^{-1}}{\Delta \omega^{2}+4 V^{2}+T_{2}^{-2}}
$$

where $\Delta \omega$ is the spectral separation between the donor and acceptor, $T_{2}^{-1}$ the homogeneous dephasing linewidth, and $V_{D A}$ is the donor-acceptor transition dipole coupling strength:

$$
V_{D A}^{2}=\kappa^{2} \frac{\mu_{D}^{2} \mu_{A}^{2}}{\mathrm{r}_{\mathrm{DA}}^{6}}
$$

Here, $\mathrm{k}^{2}$ is an orientational factor, $\mu_{D / A}$ the transition dipole moments of the donor/acceptor transitions, respectively, and $r_{D A}$ the donor-acceptor distance.

Based on the strongly nonlinear dependence of the energy transfer rate on the intermolecular distance $\left(r_{D A}^{-6}\right)$, or surface coverage $\left(c^{-3}\right)$, we can readily understand the result of Figure 7 . That is, the $40 \%$ surface coverage relative to the experiments reported in Figure 5 is expected to reduce the vibrational transfer rate by a factor $0.4^{3}=0.06$, which is too slow to be observable, in particular given the limited observation window of 30 ps due to the lower signal-to-noise ratio for a lower-coverage sample. The observations of Figure 4 to Figure 7 thus fully satisfies the theoretical expectations and furthermore indicates that neither the $\mathrm{Re}(\mathrm{CO}){ }_{3} \mathrm{Cl}$ molecules nor the Ph-dilutant molecules cluster on the surface, but rather arrange in a truly mixed monolayer. That is, if the molecules would cluster, the intermolecular distance between $\operatorname{Re}(\mathrm{CO})_{3} \mathrm{Cl}$ molecules within each one of those clusters would still be the same as in a pure $\operatorname{Re}(\mathrm{CO})_{3} \mathrm{Cl}$ sample and cross peaks should still exist. 
In our previous works, ${ }_{18}^{18}$ we observed a vibrational energy transfer rate of $\sim 90$ ps between $\operatorname{Re}(\mathrm{CO}){ }_{3} \mathrm{Cl}$ and its ${ }^{13} \mathrm{CO}$ labelled counterpart, which were co-adsorbed on an ITO surface in a $50 \% 50 \%$ mixture. Evaluating Eqs. 1 and 2 with experimentally accessible parameters for $\Delta \omega, T_{2}$ and $\mu_{D / A}$ and assuming $\mathrm{K}=1$, we had estimated an donor/acceptor distance of $\mathrm{r}_{\mathrm{DA}} \approx 4-5 \AA$, i.e., in essence a closest possible packing of $\mathrm{Re}(\mathrm{CO})_{3} \mathrm{Cl}$ molecules on the surface. Comparing these results to the system studied here, we note that the energy spacing $\Delta \omega=15 \mathrm{~cm}^{-1}$ is smaller than that upon isotope labelling $\left(\Delta \omega=46 \mathrm{~cm}^{-1}\right)$, and that the contribution of that energy spacing to Eq. 1 can already fully account for the by a factor three speed-up of the vibrational energy rate. This, in turn, implies that the dipole-dipole coupling $V_{D A}$ is about the same in both cases, which is a surprising result, since it would suggest that both samples are structurally the same, in apparent disagreement with the dimerization model of Zanni and coworkers. $^{21,22}$
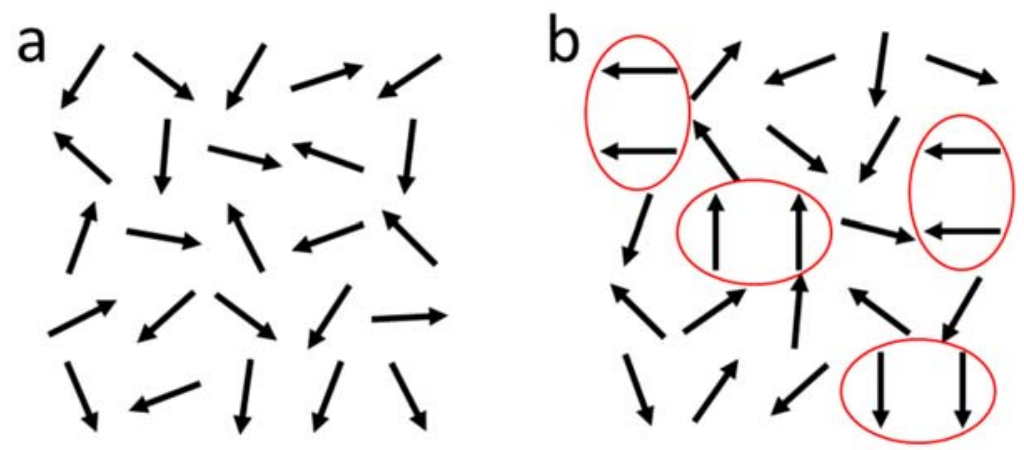

Figure 8. Sketch of the morphology of the $\mathrm{Re}(\mathrm{CO})_{3} \mathrm{Cl}$ monolayer with the arrows indicating the direction of the transition dipoles of the $A^{\prime}$ vibration. Panel (a) shows a fully disordered monolayer, and (b) one with ordered micro-domains (dimers) that are encircled in red.

Figure 8 sketches what we conceive of the morphology of the $\mathrm{Re}(\mathrm{CO})_{3} \mathrm{Cl}$ monolayer in (a) $\mathrm{MeOH}$ environment and (b) in MeCN environment. The design of that cartoon started from the question how one would distinguish "aggregated" (dimerized) molecules in the sense of the Zanni's model ${ }^{21,22}$ from "closely packed" molecules, as deduced by us from the vibrational energy transfer rate. ${ }^{18} \mathrm{An}$ apparent way to make that difference is to invoke partial order. That is, we propose that the $\operatorname{Re}(\mathrm{CO})_{3} \mathrm{Cl}$ monolayer is completely disordered in a $\mathrm{MeOH}$ environment (Figure 8 (a)), while ordered micro-domains with dimers or potentially also larger aggregates form in $\mathrm{MeCN}$. Since individual molecules are rather constraint in a closest packed monolayer, and since their mobility on the surface is expected to be very slow owing to the two anchor groups (Figure 1), it is conceivable that the transition between the two morphologies can indeed be as slow as hours (Figure 2).

With that, we return to the dipole-dipole coupling $V_{D A}$ (Eq. 2), for which we have concluded that it is essentially the same in both cases. The dominant factors in Eq. 2 are the donor-acceptor distance 
$\left(r_{D / A}\right)$ and the transition dipoles $\mu_{D / A}$, each entering in high powers. For the transition dipole moment of a dimer, it is important to realize that owing to its symmetry, only the higher frequency exciton band is bright with twice the oscillator strength, while the lower frequency exciton band is dark. ${ }^{21,22}$ The larger transition dipole would increase the vibrational energy transfer rate, but that effect is counter-balanced by the larger monomer-dimer distance due to the fact that the sheer size of a dimer is larger. That is, the effects of transition dipole and distance tend to cancel each other. The orientational factor $k$ contributes as well to the overall coupling in a complicated fashion, and we consider the fact that all these effects seem to cancel exactly a coincidence.

The discussion above separates monomers vs. dimers in a rather artificial manner, which certainly cannot be correct in a strict sense given that all molecules are closely packed. One must however understand that the 2D ATR IR spectra reflect distributions of local structures and also distributions of couplings, as emphasized by the strong inhomogeneous broadening of the 2D ATR IR spectra (Figure 2). That distribution will be broad but unimodal in a randomly distributed sample as sketched in Figure 8 (a), and will become bimodal to a certain extent when partial order is formed in the sense of Figure 8 (b), albeit still with significant spectral overlap between the two sub-bands.

\section{III.3 Alternative Explanations}

The first alternative explanation of the origin of the $2040 \mathrm{~cm}^{-1}$ band concerns the adsorption of the $\mathrm{Re}(\mathrm{CO})_{3} \mathrm{Cl}$ molecule on different surface binding sites. ${ }^{43,46}$ Given the heterogeneity of our surfaces, which exhibit a nanoparticle structure ${ }^{18}$ rather than extended crystalline terraces, there must exist a broad distribution of structurally different surface binding sites. As such, it is extremely unlikely that there exist only two dominant binding sites, at which $\operatorname{Re}(\mathrm{CO})_{3} \mathrm{Cl}$ is immobilized. In addition to this, the only way that one could rationalize the disappearance of only one of two bands upon surface dilution (Figure 4) would be the preferential binding of the Ph-dilutant to only one of the two sites. Again, we consider this as very unlikely, since both the $\operatorname{Re}(\mathrm{CO})_{3} \mathrm{Cl}$ molecule as well as the Ph-dilutant exhibit the same linker group to the surface $(\mathrm{COOH})$ and should therefore obtain a very similar surface binding configuration and stability. Finally, adsorption at different binding sites inherently depends on the lattice structure of the surface, and a change of this structure likely increases the donor-acceptor distance by more than $1 \AA$. Consequently, energy transfer, as observed in this work (Figure 5 and Figure 6), would hardly be observable between two configurations on different binding sites, or even possible crystal facets, due to the strong $\left(r^{-6}\right)$ distance dependence of the effect.

The second alternative explanation for the origin of the $2040 \mathrm{~cm}^{-1}$ band concerns the exchange of the chloride ligand in the coordination sphere of the Rhenium center with a MeCN molecule. We have also investigated the monolayer in other solvents such as, ethanol, dimethylsulfoxide, or diethylether, 
but have observed the double peak structure only in $\mathrm{MeCN}$ environment, which would support that interpretation. It is known that the replacement of negatively charged chloride ions by neutral solvent molecules results in the generation of a cationic complex of the form $\operatorname{Re}(\mathrm{CO})_{3} \mathrm{MeCN}^{+}$, which exhibits an up-shift of the $A^{\prime}(1)$ carbonyl stretching frequency due to a change of the electron density at the Rhenium atom. ${ }^{66,67}$ In such an interpretation, the band at $2025 \mathrm{~cm}^{-1}$ would correspond to the original $\mathrm{Cl}^{-}$-complex, whereas the second band $\left(2040 \mathrm{~cm}^{-1}\right)$ corresponds to the MeCN-complex. We can rule out this possibility from the absence of any $\mathrm{C} \equiv \mathrm{N}$ absorption in mesoporous ITO samples treated with $\mathrm{MeCN}$ (Fig. SI 3, $1 \mu \mathrm{m}$ thickness, to increase the signal intensity). DFT calculations on the corresponding complex (Fig. SI 1,2), as well as previous experimental reports, ${ }^{68}$ indicate that the $\mathrm{C} \equiv \mathrm{N}$ triple bond vibration of coordinated MeCN is spectrally located slightly above $2270 \mathrm{~cm}^{-1}$, thus well-separated and distinguishable from "free" MeCN $\left(2235 \mathrm{~cm}^{-1}\right)$, with appreciable oscillator strength that should easily be detectable with the signal-to-noise ratio of the spectra in Fig. SI 3.

\section{Concluding Remarks}

Despite a lot of effort, there exists no clear picture of how the solvent environment influences monolayer structures at surfaces. ${ }^{18,19,21,22,44,46,47}$ This holds particularly true for cooperative effects such as relative orientation, structural mobility and flexibility, or the formation of aggregates. The present $\mathrm{Re}(\mathrm{CO})_{3} \mathrm{Cl}$ monolayer seems to be an ideal test case to study such phenomena, given its surprisingly complex IR spectroscopy on surfaces. ${ }^{18,21,22,44,46,48}$ By systematically exchanging the solvent, a spectral feature is formed in the $A^{\prime}(1)$ symmetric stretching region of the carbonyl ligands. The process is fully reversible, which has not been observed before. With the help of control experiments, in which we modified the surface coverage of the sample molecule, we provide strong evidence that the second band is related to a dimerization of molecules at the surface, along the lines of the model proposed by Zanni and coworkers. ${ }^{21,22}$ More specifically, we suggest that the origin of this process is the solventcontrolled partial ordering of the monolayer. We observe that effect only in MeCN as solvents, and not in any other solvent such as $\mathrm{MeOH}$, ethanol, dimethylsulfoxide, or diethylether, but we can currently not explain why MeCN is special in this regard. Our observations demonstrate that even comparatively rigid, immobilized molecules exhibit a certain degree of structural flexibility that allows the adoption of ordered structures, depending on environmental conditions. We furthermore characterized in detail the dynamics of ultrafast vibrational energy transfer between monomeric and dimeric molecules, from which we can deduce a model for the morphology of the closely packed monolayer in both cases (Figure 8).

The reversible transition between ordered and disordered structures observed here takes place on the timescale of hours. Similarly slow dynamics for the overall change in the IR spectrum of Re-carbonyl 
containing monolayers of Langmuir-Blodgett films have been presented very recently, but at the same time, time-dependent cross peaks in the 2D IR spectra and have been interpreted in the framework of chemical exchange between sample sub-structures ${ }^{48}$ However, due to the non-immobilized nature of the Langmuir-Blodgett film, that sample system is highly mobile and structural flexibility on a wide range of timescales can be rationalized. Taking both studies together, there exist now growing evidence for very slow structural transitions in monolayer samples at different types of interfaces, whether the molecules are immobilization covalently or not. In this regard, it will be interesting to see to what extent the ultrafast and ultraslow dynamics of different samples can be correlated and manipulated, for instance by temperature and pressure variations.

Finally, we set our results in a broader context of heterogeneous chemistry. The observation of intermolecular interactions between adsorbates such as the ones described here is of significant importance for a broad range of applications for instance in heterogeneous catalysis ${ }^{2,3,5,7}$, molecular recognition ${ }^{69-71}$, chemical sensing ${ }^{72-74}$ or dye sensitized solar cells ${ }^{26,27,75-79}$. In the context of the latter, dye aggregation is a topic that was discussed extensively, since it is believed to limit the performance of these devices. Dye molecules with bulky side groups have been synthesized to minimize aggregation, but the present study suggests that also the effect of the solvent needs to be taken into account. Thinking in another direction, metal-carbonyl complexes similar to the ones investigated here, which perform as $\mathrm{CO}_{2}$-reduction catalysts, have been proposed to form dimerized intermediates upon electrochemical treatment. ${ }^{23-25}$ If the sample molecules are immobilized, then structural flexibility and the possibility to dimerize even at the surface are then expected to directly influence for instance a catalytic performance of the complexes. For all of these applications, intermolecular dynamics on different timescales, i.e. ultrafast to macroscopic, are largely unexplored to date, since femtosecond multi-dimensional spectroscopy on surfaces became available only recently. ${ }^{19,28,31,80,81}$ With the development of these techniques, cooperative effects as well as structural properties (e.g. intermolecular distances) can now be investigated.

Supporting Information: Quantum-chemistry calculations of $\operatorname{Re}(\mathrm{CO})_{3} \mathrm{Cl}$ and $\operatorname{Re}(\mathrm{CO})_{3} \mathrm{MeCN}$ as well as FTIR spectra of $\mathrm{Re}(\mathrm{CO})_{3} \mathrm{Cl}$ on ITO after incubation in $\mathrm{MeCN}$ in the spectral range of the expected $\mathrm{CN}$ vibration, should the ligand exchange. This information is available free of charge via the Internet at http://pubs.acs.org.

\section{Acknowledgements}

We thank Martin T. Zanni, Laurent Sévery, Thomas Moehl, Mathias Mosberger, Roger Alberto, RicardoFernandez-Teran, Jan Helbing, Feng Shao, Liqing Zheng, Renato Zenobi, Jürg Hutter and Marcella 
lannuzzi-Mauri for insightful discussions, and Rolf Pfister for the synthesis of the investigated samples.

The research was funded by the Swiss National Science Foundation through grant number CRSII2_160801/1 and by the URRP LightChEC of the University of Zurich. Experimental support by the Center of Microscopy and Imaging at the University of Zurich (ZMB) with regard to the preparation and characterization of the substrate layers is gratefully acknowledged.

\section{Corresponding Authors}

*philip.kraack@gmail.com

Phone: +41446354477

Fax: +41446356838

†peter.hamm@chem.uzh.ch

Phone: +41446354431

Fax: +41446356838

\section{References}

(1) Fox, M. A.; Dulay, M. T. Heterogeneous Photocatalysis. Chem. Rev. 1993, 93, 341-357.

(2) Somorjai, G. A.; Contreras, A. M.; Montano, M.; Rioux, R. M. Clusters, Surfaces and Catalysis. Proc. Natl. Acad. Sci. U. S. A. 2006, 103, 10577-10583.

(3) Arakawa, H.; Aresta, M.; Armor, J. N.; Barteau, M. A.; Beckman, E. J.; Bell, A. T.; Bercaw, J. E.; Creutz, C.; Dinjus, E.; Dixon, D. A.; et al. Catalysis Research of Relevance to Carbon Management: Progress, Challenges, and Opportunities. Chem. Rev. 2001, 101, 953-996.

(4) Bailey, D. C.; Langer, S. H. Immobilized Transition-Metal Carbonyls and Related Catalysts. Chem. Rev. 1981, 81, 110-144.

(5) Schneider, J.; Matsuoka, M.; Takeuchi, M.; Zhang, J.; Horiuchi, Y.; Anpo, M.; Bahnemann, D. W. Understanding TiO2 Photocatalysis : Mechanisms and Materials. Chem. Rev. 2014, 114, 99199986.

(6) Bahnemann, D. Photocatalytic Water Treatment: Solar Energy Applications. Sol. Energy 2004, 77, 445-459.

(7) Grätzel, M. Energy Resources through Photochemistry and Catalysis; Grätzel, M., Ed.; Academic Press, Inc.: New York, 1983.

(8) Kumar, B.; Llorente, M.; Froehlich, J.; Dang, T.; Sathrum, A.; Kubiak, C. P. Photochemical and Photoelectrochemical Reduction of CO2. Annu. Rev. Phys. Chem. 2012, 63, 541-569.

(9) Centi, G.; Perathoner, S. Opportunities and Prospects in the Chemical Recycling of Carbon Dioxide to Fuels. Catal. Today 2009, 148, 191-205.

(10) Crabtree, R. H. Electrochemical and Photoelectrochemical Conversion of CO2 to Alcohols; John Wiley \& Sons, Ltd.: New York, 2010. 
(11) Aresta, M. Carbon Dioxide as Chemical Feedstock; John Wiley \& Sons, 2010.

(12) Hou, W.; Hung, W. H.; Pavaskar, P.; Goeppert, A.; Aykol, M.; Cronin, S. B. Photocatalytic Conversion of $\mathrm{CO} 2$ to Hydrocarbon Fuels via Plasmon-Enhanced Absorption and Metallic Interband Transitions. ACS Catal. 2011, 1, 929-936.

(13) Kuhl, K. P.; Hatsukade, T.; Cave, E. R.; Abram, D. N.; Kibsgaard, J.; Jaramillo, T. F. Electrocatalytic Conversion of Carbon Dioxide to Methane and Methanol on Transition Metal Surfaces. J. Am. Chem. Soc. 2014, 136, 14107-14113.

(14) Fujita, E. Photochemical Carbon Dioxide Reduction with Metal Complexes. Coord. Chem. Rev. 1999, 185-186, 373-384.

(15) Sahara, G.; Ishitani, O. Efficient Photocatalysts for CO2 Reduction. Inorg. Chem. 2015, 54, 5096-5104.

(16) Costamagna, J.; Ferraudi, G.; Canales, J.; Vargas, J. Carbon Dioxide Activation by AzaMacrocyclic Complexes. Coord. Chem. Rev. 1996, 148, 221-248.

(17) Love, J. C.; Estroff, L. A.; Kriebel, J. K.; Nuzzo, R. G.; Whitesides, G. M. Self-Assembled Monolayers of Thiolates on Metals as a Form of Nanotechnology. Chem. Rev. 2005, 105, 11031169.

(18) Kraack, J. P.; Frei, A.; Alberto, R.; Hamm, P. Ultrafast Vibrational Energy-Transfer in Catalytic Monolayers at Solid-Liquid Interfaces. J. Phys. Chem. Lett. 2017, 8, 2489-2495.

(19) Rosenfeld, D. E.; Gengeliczki, Z.; Smith, B. J.; Stack, T. D. P.; Fayer, M. D. Structural Dynamics of a Catalytic Monolayer Probed by Ultrafast 2D IR Vibrational Echoes. Science 2011, 334, 634639.

(20) Ulman, A. Formation and Structure of Self-Assembled Monolayers. Chem. Rev. 1996, 96, 15331554.

(21) Oudenhoven, T. A.; Joo, Y.; Laaser, J. E.; Gopalan, P.; Zanni, M. T. Dye Aggregation Identified by Vibrational Coupling Using 2D IR Spectroscopy. J. Chem. Phys. 2015, 142, 212449.

(22) Laaser, J. E.; Christianson, J. R.; Oudenhoven, T. A.; Joo, Y.; Gopalan, P.; Schmidt, J. R.; Zanni, M. T. Dye Self-Association Identified by Intermolecular Couplings between Vibrational Modes as Revealed by Infrared Spectroscopy, and Implications for Electron Injection. J. Phys. Chem. C 2014, 118, 5854-5861.

(23) Machan, C. W.; Sampson, M. D.; Chabolla, S. A.; Kubiak, C. P. Developing a Mechanistic Understanding of Molecular Electrocatalysts for $\mathrm{CO} 2$ Reduction Using Infrared Spectroelectrochemistry. Organometallics 2014, 33, 4550-4559.

(24) Neri, G.; Donaldson, P. M.; Cowan, A. J. The Role of Electrode-Catalyst Interactions in Enabling Efficient $\mathrm{CO} 2$ Reduction with $\mathrm{Mo}(\mathrm{bpy})(\mathrm{CO}) 4$ As Revealed by Vibrational Sum-Frequency Generation Spectroscopy. J. Am. Chem. Soc. 2017, 139, 13791-13797.

(25) Bourrez, M.; Molton, F.; Chardon-Noblat, S.; Deronzier, A. [Mn(bipyridyl)(CO)3Br]: An Abundant Metal Carbonyl Complex as Efficient Electrocatalyst for CO2 Reduction. Angew. Chemie - Int. Ed. 2011, 50, 9903-9906.

(26) Hagfeldt, A.; Boschloo, G.; Sun, L.; Kloo, L.; Pettersson, H. Dye-Sensitized Solar Cells. Chem. Rev. 2010, 110, 6595-6663.

(27) Ning, Z.; Fu, Y.; Tian, H. Improvement of Dye-Sensitized Solar Cells: What We Know and What We Need to Know. Energy Environ. Sci. 2010, 3, 1170-1181.

(28) Kraack, J. P. J. P.; Lotti, D.; Hamm, P. Ultrafast, Multidimensional Attenuated Total Reflectance Spectroscopy of Adsorbates at Metal Surfaces. J. Phys. Chem. Lett. 2014, 5, 2325-2329. 
(29) Kraack, J. P.; Lotti, D.; Hamm, P. 2D Attenuated Total Reflectance Infrared Spectroscopy Reveals Ultrafast Vibrational Dynamics of Organic Monolayers at Metal-Liquid Interfaces. J. Chem. Phys. 2015, 142, 212413.

(30) Kraack, J. P.; Lotti, D.; Hamm, P. Surface-Enhanced, Multi-Dimensional Attenuated Total Reflectance Spectroscopy. Proc. SPIE, Phys. Chem. Interfaces Nanomater. XIV 2015, 9549, 95490S.

(31) Lotti, D.; Hamm, P.; Kraack, J. P. Surface-Sensitive Spectro-Electrochemistry Using Ultrafast 2D ATR IR Spectroscopy. J. Phys. Chem. C 2016, 120, 2883-2892.

(32) Kraack, J. P.; Kaech, A.; Hamm, P. Surface Enhancement in Ultrafast 2D ATR IR Spectroscopy at the Metal-Liquid Interface. J. Phys. Chem. C 2016, 120, 3350-3359.

(33) Kraack, J. P.; Hamm, P. Vibrational Ladder-Climbing in Surface-Enhanced, Ultrafast Infrared Spectroscopy. Phys. Chem. Chem. Phys. 2016, 18, 16088-16093.

(34) Kraack, J. P.; Hamm, P. Surface-Sensitive and Surface-Specific Ultrafast Two-Dimensional Vibrational Spectroscopy. Chem. Rev. 2016, 117, 10623-10664.

(35) Hamm, P.; Zanni, M. T. Concepts and Methods of 2D Infrared Spectroscopy; Cambridge University Press: Cambridge, 2011.

(36) Fayer, M. D. Ultrafast Infrared Vibrational Spectroscopy; Fayer, M. D., Ed.; CRC Press: Boca Raton, London, New York, 2013.

(37) Cho, M. Two-Dimensional Optical Spectroscopy; CRC Press: Boca Raton, 2009.

(38) Cho, M. Coherent Two-Dimensional Optical Spectroscopy. Chem. Rev. 2008, 108, 1331-1418.

(39) Bürgi, T.; Baiker, A. Attenuated Total Reflection Infrared Spectroscopy of Solid Catalysts Functioning in the Presence of. Adv. Catal. 2006, 50, 227-283.

(40) Andanson, J.-M.; Baiker, A. Exploring Catalytic Solid/liquid Interfaces by in Situ Attenuated Total Reflection Infrared Spectroscopy. Chem. Soc. Rev. 2010, 39, 4571-4584.

(41) Mojet, B. L.; Ebbesen, S. D.; Lefferts, L. Light at the Interface: The Potential of Attenuated Total Reflection Infrared Spectroscopy for Understanding Heterogeneous Catalysis in Water. Chem. Soc. Rev. 2010, 39, 4643-4655.

(42) Windle, C. D.; Perutz, R. N. Advances in Molecular Photocatalytic and Electrocatalytic CO 2 Reduction. Coord. Chem. Rev. 2012, 256, 2562-2570.

(43) Calabrese, C.; Vanselous, H.; Petersen, P. B. Deconstructing the Heterogeneity of SurfaceBound Catalysts: Rutile Surface Structure Affects Molecular Properties. J. Phys. Chem. C 2016, 120, 1515-1522.

(44) Rosenfeld, D. E.; Nishida, J.; Yan, C.; Kumar, S. K. K.; Tamimi, A.; Fayer, M. D. Structural Dynamics at Monolayer-Liquid Interfaces Probed by 2D IR Spectroscopy. J. Phys. Chem. C 2013, 117, 1409-1420.

(45) Nishida, J.; Yan, C.; Fayer, M. D. Dynamics of Molecular Monolayers with Different Chain Lengths in Air and Solvents Probed by Ultrafast 2D IR Spectroscopy. J. Phys. Chem. C 2014, 118, 523-532.

(46) Xiong, W.; Laaser, J. E.; Paoprasert, P.; Franking, R. A.; Hamers, R. J.; Gopalan, P.; Zanni, M. T. Transient 2D IR Spectroscopy of Charge Injection in Dye-Sensitized Nanocrystalline Thin Films. J. Am. Chem Soc. 2009, 131, 18040-18041.

(47) Yan, C.; Yuan, R.; Nishida, J.; Fayer, M. D. Structural Influences on the Fast Dynamics of Alkylsiloxane Monolayers on SiO 2 Surfaces Measured with 2D IR Spectroscopy. J. Phys. Chem. C 2015, 119, 16811-16823. 
(48) Yan, C.; Thomaz, J. E.; Wang, Y.-L.; Nishida, J.; Yuan, R.; Breen, J. P.; Fayer, M. D. Ultrafast to Ultraslow Dynamics of a Langmuir Monolayer at the Air/Water Interface Observed with Reflection Enhanced 2D IR Spectroscopy. J. Am. Chem. Soc. 2017, 139, 16518-16527.

(49) Helbing, J.; Hamm, P. Compact Implementation of Fourier Transform Two-Dimensional IR Spectroscopy without Phase Ambiguity. J. Opt. Soc. Am B 2010, 28, 171-178.

(50) Kraack, J. P. Ultrafast Structural Molecular Dynamics Investigated with 2D Infrared Spectroscopies. Top Curr Chem 2017, 375:86, 1-93.

(51) Kwak, K.; Zheng, J.; Cang, H.; Fayer, M. D. Ultrafast Two-Dimensional Infrared Vibrational Echo Chemical Exchage Experiments and Theory. J. Phys. Chem. B 2006, 110 (40), 19998-20013.

(52) Woutersen, S.; Mu, Y.; Stock, G.; Hamm, P. Hydrogen-Bond Lifetime Measured by TimeResolved 2D-IR Spectroscopy: N-Methylacetamide in Methanol. Chem. Phys. 2001, 266, 137147.

(53) Anfuso, C. L.; Ricks, A. M.; Rodr, W.; Lian, T. Ultrafast Vibrational Relaxation Dynamics of a Rhenium Bipyridyl. J. Phys. Chem. C 2012, 116, 26377-26384.

(54) Ricks, A. M.; Anfuso, C. L.; Rodríguez-Córdoba, W.; Lian, T. Vibrational Relaxation Dynamics of Catalysts on TiO2 Rutile Single Crystal Surfaces and Anatase Nanoporous Thin Films. Chem. Phys. 2013, 422, 264-271.

(55) Delor, M.; Sazanovich, I. V.; Towrie, M.; Spall, S. J.; Keane, T.; Blake, A. J.; Wilson, C.; Meijer, A. J. H. M.; Weinstein, J. A. Dynamics of Ground and Excited State Vibrational Relaxation and Energy Transfer in Transition Metal Carbonyls. J. Phys. Chem. B 2014, 118, 11781-11791.

(56) Ge, A.; Rudshteyn, B.; Zhu, J.; Maurer, R. J.; Batista, V. S.; Lian, T. Electron-Hole-Pair-Induced Vibrational Energy Relaxation of Rhenium Catalysts on Gold Surfaces. J. Phys. Chem. Lett. 2017, DOI: 10.1021/acs.jpclett.7b02885.

(57) Mirkovic, T.; Ostroumov, E. E.; Anna, J. M.; van Grondelle, R.; Govindjee; Scholes, G. D. Light Absorption and Energy Transfer in the Antenna Complexes of Photosynthetic Organisms. Chem. Rev. 2017, 117, 249-293.

(58) Scholes, G. D. Long-Range Resonance Energy Transfer in Molecular Systems. Annu. Rev. Phys. Chem. 2003, 54, 57-87.

(59) Chenu, A.; Scholes, G. D. Coherence in Energy Transfer and Photosynthesis. Annu. Rev. Phys. Chem. 2014, 66, 69-96.

(60) Neuhaus, D.; Williamson, M. P. The Nuclear Overhauser Effect in Structural and Conformational Analysis; VCH New York: New York, 1989.

(61) van der Meer, B. W. Förster Theory. In FRET - Förster Resonance Energy Transfer; Wiley-VCH Verlag GmbH \& Co. KGaA, 2013; pp 23-62.

(62) Li, J.; Yuan, K.; Chen, H.; Miranda, A.; Shen, Y.; Jiang, B.; Chen, Y.; Zhang, Y.; Guo, X.; Zheng, J. Nonresonant Vibrational Energy Transfer on Metal Nanoparticle/Liquid Interface. J. Phys. Chem. C 2016, 19, 25173-25179.

(63) Chen, H.; Wen, X.; Zhang, J.; Wu, T.; Gong, Y.; Zhang, X.; Yuan, J.; Yi, C.; Lou, J.; Ajayan, P. M.; et al. Ultrafast Formation of Interlayer Hot Excitons in Atomically Thin MoS2/WS2 Heterostructures. Nat. Commun. 2016, 7, 12512.

(64) Chen, H.; Wen, X.; Guo, X.; Zheng, J. Intermolecular Vibrational Energy Transfers in Liquids and Solids. Phys. Chem. Chem. Phys. 2014, 16, 13995-14014.

(65) Chen, H.; Bian, H.; Li, J.; Wen, X.; Zheng, J. Ultrafast Multiple-Mode Multiple-Dimensional Vibrational Spectroscopy. Int. Rev. Phys. Chem. 2012, 31, 469-565. 
(66) Hevia, E.; Pérez, J.; Riera, V.; Miguel, D.; Kassel, S.; Rheingold, A. New Synthetic Routes to Cationic Rhenium Tricarbonyl Bipyridine Complexes with Labile Ligands. Inorg. Chem. 2002, 41, 4673-4679.

(67) Hevia, E.; Pe, J.; Miguel, D. New Octahedral Rhenium (I) Tricarbonyl Amido Complexes. 2002, 21, 1966-1974.

(68) Christensen, P.; Hamnett, A.; Muir, A. V. G.; Timney, J. A. An in Situ Infrared Study of CO, Reduction Catalysed by Rhenium Tricarbonyl Bipyridyl Derivatives. J. Chem. Soc. Dalt. Trans 1992, 9, 1455-1463.

(69) Leblanc, R. M. Molecular Recognition at Langmuir Monolayers. Curr. Opin. Chem. Biol. 2006, 10, 529-536.

(70) Spinke, J.; Liley, M.; Angermaierj, H. G. L.; Knoll, W. Molecular Recognition at Self -Assembled Monolayers : The Construction of Multicomponent Multilayers. Langmuir 1993, 9, 1821-1825.

(71) Sampson, N. S.; Mrksich, M.; Bertozzi, C. R. Surface Molecular Recognition. Proc. Nat. Acad. Sci. USA 2000, 98, 2000-2001.

(72) Kellner, R.; Mizaikoff, B.; Jakusch, M.; Wanzenböck, H. D.; Weissenbacher, N. Surface-Enhanced Vibrational Spectroscopy: A New Tool in Chemical IR Sensing? Appl. Spectrosc. 1997, 51, 495503.

(73) Hoang, C. V; Oyama, M.; Saito, O.; Aono, M.; Nagao, T. Monitoring the Presence of Ionic Mercury in Environmental Water by Plasmon-Enhanced Infrared Spectroscopy. Sci. Rep. 2013, 3, 1175.

(74) Kim, I.; Kihm, K. Nano Sensing and Energy Conversion Using Surface Plasmon Resonance (SPR). Materials (Basel). 2015, 8, 4332-4343.

(75) Polo, A. S.; Itokazu, M. K.; Murakami Iha, N. Y. Metal Complex Sensitizers in Dye-Sensitized Solar Cells. Coord. Chem. Rev. 2004, 248, 1343-1361.

(76) Hagfeldt, A.; Grätzel, M. Molecular Photovoltaics. Acc. Chem. Res. 2000, 33, 269-277.

(77) Clifford, J. N.; Martínez-Ferrero, E.; Viterisi, A.; Palomares, E. Sensitizer Molecular StructureDevice Efficiency Relationship in Dye Sensitized Solar Cells. Chem. Soc. Rev. 2011, 40, 16351646 .

(78) Yu, Z.; Li, F.; Sun, L. Recent Advances in Dye-Sensitized Photoelectrochemical Cells for Solar Hydrogen Production Based on Molecular Components. Energy Environ. Sci. 2014, 8, 760-775.

(79) Kalyanasundaram, K. Applications of Functionalized Transition Metal Complexes in Photonic and Optoelectronic Devices. Coord. Chem. Rev. 1998, 177, 347-414.

(80) Xiong, W.; Laaser, J. E.; Mehlenbacher, R. D.; Zanni, M. T. Adding a Dimension to the Infrared Spectra of Interfaces Using Heterodyne Detected 2D Sum- Frequency Generation ( HD 2D SFG ) Spectroscopy. Proc. Nat. Acad. Sci. USA 2011, 108, 20902-20907.

(81) Yan, C.; Yuan, R.; Pfalzgraff, W. C.; Nishida, J.; Wang, L.; Markland, T. E.; Fayer, M. D. Unraveling the Dynamics and Structure of Functionalized Self-Assembled Monolayers on Gold Using 2D IR Spectroscopy and MD Simulations. Proc. Natl. Acad. Sci. 2016, 113, 4929-4934. 
TOC Graphic

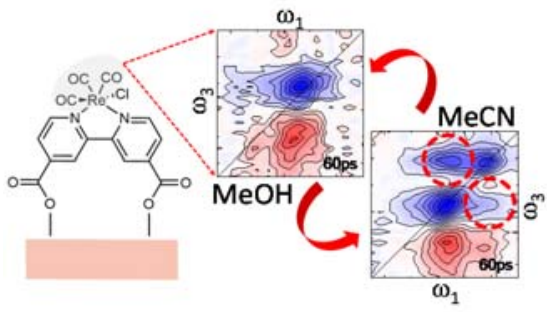

